\title{
RETRACTED: Optimization method of emergency logistics network in the initial post-earthquake stage considering multiple factors and the case analysis
}

Junxiang $\mathrm{Xu}^{\mathrm{a}, *}$, Jingni Guo ${ }^{\mathrm{a}}$, Jin Zhang ${ }^{\mathrm{a}, \mathrm{b}, \mathrm{c}}$, Yongdong Sun ${ }^{\mathrm{a}}$, Weihua Liu ${ }^{\mathrm{d}}$ and Hui $\mathrm{Ma}^{\mathrm{d}}$ ${ }^{a}$ School of Transportation and Logistics, Southwest Jiaotong University, Chengdu, China

${ }^{\mathrm{b}}$ National United Engineering Laboratory of Integrated and Intelligent Transportation,

Southwest Jiaotong University, Chengdu, China

${ }^{\mathrm{c}}$ National Engineering Laboratory of Integrated Transportation Big Data Application Technology,

Chengdu, China

${ }^{\mathrm{d} C h i n a ~ R a i l w a y ~ N o . ~} 2$ Engineering Group Co., LTD, Chengdu, China

This article has been retracted, and the online PDF has been watermarked "RETRACTED". The retraction notice is available at http://doi.org/10.3233/IFS-219216.

\section{Introduction}

The outbreak of a large-scale earthquake may cause many casualties and a large demand for disaster relief supplies in a short time. The initial stage especially the first 72 hours after the earthquake is the prime time for emergency rescue. During this period, the demand for emergency supplies such as medicine,

${ }^{*}$ Corresponding author. Junxiang $\mathrm{Xu}$, School of Transportation and Logistics, Southwest Jiaotong University, Chengdu 611756, China. E-mail: 1933348984@qq.com. blood, and rescue tools is very urgent. Therefore, after a major earthquake, an effective emergency logistics network must be established immediately to realize the rapid allocation of emergency supplies from the supply points to the disaster-stricken points.

A large number of researchers have described the optimization design problem of an emergency logistics network after the earthquake as a facility location-allocation problem (FLAP). For example, Salman et al. [1] studied emergency FLAP under random road network damage. Boonmee et al. [2] 
reviewed facility location problem (FLP) in humanitarian relief logistics. Tzeng et al. [3] considered the characteristics of emergency material allocation after the earthquake and studied the multi-objective location (LAP) model of emergency transit facilities. Xiong et al. [4] aimed to maximize the satisfaction of transportation time and the fairness of material distribution, based on the two-level programming method. They studied the location-intermodal problem in the emergency logistics system after the earthquake, and designed a corresponding hybrid genetic algorithm. Zhang et al. [5] considered the demand uncertainty and studied the emergency FLAP in the initial postearthquake stage and described it as a random hybrid integer programming model. Yahyaei and BozorgiAmiri [6] studied the integrated location selection of emergency material supply facilities and refuge facilities after the earthquake.

The above studies all contain a hidden assumption, that is, the facility is completely reliable without disruption, but this assumption does not conform to real situation [7]. Due to natural disasters or human factors, the established facilities may be interrupted. Once the facility is interrupted, the point that wants the service may not be supplied with materials, or other facilities need to be reassigned to serve it. Other temporarily assigned facilities are farther away and may not have sufficient inventory capacity. Therefore, the phenomenon of facility disruption will affect the operating efficiency of the entire logistics system. Mishra et al. [8] used a two-stage stochastic programming method to study the supply chain optimization design problem considering the risk of disruption, uncertain demand for multiple products and uncertain freight rates. The results showed that the model considering the risk factors could save more than 5\% cost. Therefore, it is necessary to consider the risk of facility disruption, which will help build a reliable logistics network and reduce emergency costs. Snyder et al. [9] was the first to introduce disruption risk and studied the reliability facility location problem (RFLP). They constructed two types of RFLP models in [9], namely the reliability uncapacitated fixedcharge location problem (RUFLP) and the reliability p-median problem (RPMP). Their model considered both the daily operating costs and the expected disruption cost of the facility. They proved that a small increase in cost could greatly improve the reliability of the system. After more than ten years, RFLP has received great attention from researchers and has become a frontier problem in location selection research, and has achieved great research results. An et al. [10] and Albareda-Sambola et al. [11] also studied RPMP and RUFLP, respectively. In addition, Sadeghi et al. [12] studied the reliability fixed cost location problem with capacity constraints. Aydin et al. [13] studied the reliability p-hub coverage location problem with capacity constraints.

Traditional RFLP research generally focuses on the conventional environment, and there is rare research on the location of emergency facilities considering the risk of disruption. Zhou et al. [14] took the blood station as the research object, and considered the timeliness and reliability of blood product supply, and studied the RPMP model with different failure probabilities of facilities. Fu et al. [15] established an RFLP model for a regional emergency reserve facility. The research objects of the above-mentioned references are emergency reserve facilities in the process of disaster prevention and preparedness, and system costs usually need to be considered. The supply of emergency materials in the initial post-earthquake stage has weak property of cost and other typical characteristics, so these research results of RFLP for disaster prevention and emergency response are difficult to be applied tolocation and allocation decisions during the post-disaster rescue period.

The allocation of emergency supplies in the initial stage of post-earthquake rescue has many outstanding features, which are described as follows. (1) Uncertain demand. Due to the lack of information transmission channels in the initial post-earthquake stage and the unclear information on the disaster situation, the demand information in the disaster-stricken area can only be roughly inferred based on the factors such as earthquake intensity, the earthquake resistance level of buildings, the time of earthquake, and the population density of disaster-stricken area. (2) There are many types of disaster relief materials and high urgency. During this period, a variety of emergency supplies such as food, drinking water, tents, and medicines need to be transported urgently. Due to the serious disaster in the initial stage, the demand for materials is of high urgency. (3) The road capacity is severely restricted. After a large-scale earthquake, the road network is damaged and it is difficult to completely repair it in a short period of time, reducing the transportation efficiency. (4) Demand greatly exceeds supply. In the initial post-earthquake stage, the explosive demand for emergency supplies, limited supplies and financing in the short term, coupled with the slowdown in the transportation of damaged materials on the road network, lead to a shortage of supplies during this period. (5) It is difficult to accurately obtain 
the facility disruption probabilities. At this time, it is more practical to describe the problem based on the disruption scenario.

In response to such problems, this paper introduces facility disruption scenarios based on traditional RFLP and emergency FLAP, considers the characteristics of emergency material allocation, to research the location-allocation problem in the optimization design of emergency logistics network in the initial stage of post-earthquake rescue. The corresponding genetics algorithm is designed to solve the problem. The rest of this paper is arranged as follows. Section 2 is modeling method, including problem description, symbols and variables, mathematic model and fuzzy parameter processing. Section 3 introduces the algorithm design, and a hybrid genetic algorithm with integer coding is designed. Section 4 shows a case analysis. Section 5 discusses the conclusions and further research prospects.

\section{Model research}

\subsection{Problem description}

In order to quickly respond to emergency needs in the initial stage of post-earthquake rescue (1-7 days after the earthquake), it is necessary to quickly build an emergency logistics network. The core is to reasonably determine the location of emergency material distribution center. The candidate distribution center has capacity limitations. A transit facility is responsible for receiving rescue materials from the higher-level distribution points and quickly distributing various emergency materials to various disaster-stricken points. The primary goal of emergency rescue in the initial post-earthquake stage is to quickly respond to the needs of the disaster-stricken area. Therefore, the best timeliness of emergency supplies should be the optimization goal. Due to the shortage of many types of materials during this period, the fairness of the distribution of emergency materials has also become a major concern of the unsatisfied rates of any two disaster-stricken points does not exceed a given threshold.

The demand for disaster relief materials is uncertain. According to the characteristics of the demand for materials after the earthquake, the uncertain demand is treated as a triangular fuzzy number. At the same time, the roads affected by the earthquake are severely damaged during the decision-making period, and the speed of vehicles is limited. In this paper, road condition coefficients are introduced to reflect the actual speed of vehicles. Due to the threat of aftershocks, landslides and other factors, the distribution center established may fail. In view of the fact that it is difficult to accurately obtain the specific probability of facility disruption, a number of typical disruption scenarios are selected for modeling.

In summary, the problem can be described as a FLAP model that considers facility disruption scenarios, fuzzy demands, multiple types of materials, and capacity constraints. The key problem to be solved is how many distribution centers need to be established? where are they built? how to determine the assignment relationship between the disaster-stricken points and the distribution center? How many materials should be allocated to each disaster-stricken point?

\subsection{Symbols and variables}

The assumptions are as follows. (1) The location of the disaster-stricken point is known; (2) The location of each candidate distribution center is known and has processing capacity limitations; (3) Each distribution center can serve multiple disaster-stricken points at the same time; each disaster-stricken point is only assigned to an open and uninterrupted distribution center for emergency supplies.

The symbols are defined as follows:

(1) parameter

$I$ : set of disaster-stricken points, $i \in I$;

$J$ : set of candidate emergency supplies distribution centers, $j \in J$

$C$ : set of types of emergency supplies, $c \in C$;

$W:$ combination of scenarios, $w \in W$;

$$
a_{j w}=\left\{\begin{array}{l}
0, \text { emergency logistics distribution center } j \text { is interrupted under scenario } w \\
1, \text { otherwise }
\end{array}\right.
$$

victims. In order to ensure the fairness of the distribution of emergency supplies among disaster-stricken points, it is required that the difference between the $d_{i j}$ : distance from distribution center $j$ to disasterstricken point $I$

$v_{i j}$ : the transportation speed from distribution center $j$ to disaster-stricken point $i$ under normal conditions; 
$\gamma_{i j}$ : the road condition coefficient from distribution center $j$ to disaster-stricken point $i, 0 \leq \gamma_{i j} \leq 1$;

$T_{i j}$ : transportation time from distribution center $j$ to disaster-stricken point $i$ under the condition of damaged road network;

$W_{j}$ : maximum processing capacity of distribution center $j$ during the decision-making period;

$\tilde{D}_{i c}: \tilde{D}_{i c}=\left(D_{i c}^{1}, D_{i c}^{2}, D_{i c}^{3}\right)$ the demand for $c$-type material at disaster-stricken point $i$ can be described as a triangular fuzzy number $\tilde{D}_{i c}=\left(D_{i c}^{1}, D_{i c}^{2}, D_{i c}^{3}\right)$;

$K Q_{c}$ : maximum available amount of $c$-type emergency supplies;

$q_{i j}$ : The largest single capacity from distribution center $j$ to disaster-stricken point $I$;

$u_{i w}$ : The unsatisfied rate of the demand at disaster-stricken point $i$ under scenario $w$;

$\xi$ : a small positive number.

(2) Decision variables

$Q_{i c w}$ : under scenario $w$, the distribution amount of $c$-type materials at disaster-stricken point $I$;

$Q_{j c}$ : the supply amount of $c$-type materials by emergency supplies distribution center $j$;

$$
\begin{gathered}
X_{j}=\left\{\begin{array}{l}
1, \text { establish a distribution center at point } j \\
0, \text { otherwise }
\end{array}\right. \\
Y_{i j}=\left\{\begin{array}{l}
1, \text { distribution center } j \text { provides service for demand point } i \\
0, \text { otherwise }
\end{array}\right. \\
Y_{i^{\prime} j}=\left\{\begin{array}{l}
1, \text { when the initial assigned facility } j \text { of demand point } i \text { is interrupted and } \\
\text { re - assigned to the latest open and uninterrupted facility } j \\
0, \text { otherwise }
\end{array}\right.
\end{gathered}
$$

\subsection{Mathematic model}

According to the problem description and assumptions, the mathematical model is established as follows.

$$
\begin{gathered}
Z=\min \max _{w \in W} \sum_{j}\left(\sum_{i}\left[\frac{\sum_{c} Q_{i c w}}{q_{i j}}\right] T_{i j} Y_{i j}+\sum_{i^{\prime}}\left[\frac{\sum_{c} Q_{i^{\prime} c w}}{q_{i^{\prime} j}}\right] T_{i^{\prime} j} Y_{i^{\prime} j}\right) a_{j w} \\
Y_{i j} \leq X_{j}, \forall i \in I, j \in J \\
\sum_{i} Y_{i j} \leq 1, \forall j \in J \\
Y_{i^{\prime} j}=\left\{\begin{array}{c}
1, t_{i^{\prime} j}=\min _{j_{0} \in J} t_{i^{\prime} j}, X_{j} a_{j} X_{j_{0}} \alpha_{j_{0}} Y_{i^{\prime} j^{\prime}}=1 \text { and } a_{j^{\prime}}=0 \\
0, \text { otherwise }
\end{array}, \forall i \in I, j \in J\right. \\
Q_{j c}=\sum_{i} \tilde{D}_{i c} Y_{i j} ; \forall j \in J, c \in C
\end{gathered}
$$




$$
\begin{aligned}
& Q_{i c w}=\frac{Q_{j c}}{\sum_{i} \tilde{D}_{i c} Y_{i j}+\sum_{i^{\prime}} \tilde{D}_{i^{\prime} c} Y_{i^{\prime} j}} D_{i c}, \forall i \in I, c \in C, w \in W, \text { and } Y_{i j} a_{j w}=1 \\
& i_{i w}=\left\{\begin{array}{l}
\frac{\sum_{c}\left(\tilde{D}_{i c}-Q_{i c w}\right)}{\sum_{c} \tilde{D}_{i c}}, \tilde{D}_{i c}>Q_{i c w} \\
1, \tilde{D}_{i c} \leq Q_{i c w}
\end{array}, \forall i \in I, w \in W\right. \\
& \left|u_{e w}-u_{f w}\right| \leq \xi, \forall e \in I, f \in I \text { and } e \neq f, w \in W \\
& \sum_{J} Q_{j c} \leq K Q_{c}, \forall c \in C \\
& \sum_{e} Q_{j e} \leq W_{j}, \forall j \in J \\
& Q_{j c}, Q_{i c w} \text { are positive integers, } \forall i \in I, j \in J, c \in C, w \in W \\
& X_{j}, Y_{i j} \in\{0,1\}, \forall i \in I, j \in J
\end{aligned}
$$

Eq. (1) indicates that the maximum delivery time in any scenario is the smallest, thereby ensuring the timeliness of the emergency logistics network. The first term of Eq. (1) represents the delivery time of materials received by disaster-stricken points where the initial designated facility has not been interrupted, and the second term represents the delivery time of materials received by disaster-stricken points where the initially designated facility has been interrupted. Eq. (2) means that the premise for the distribution center to provide services is that the facilities are open and uninterrupted; Eq. (3) means that each disaster-stricken point is only assigned to one distribution center; Eq. (4) means that the road network is damaged. The transportation time from the distribution center to the disaster-stricken point is as follows; Eq. (5) indicates that if the distribution center initially assigned by the disaster-stricken point is interrupted, it will be reassigned to the nearest open and uninterrupted facility; Eq. (6) is the material supply constraint in the distribution of emergency supplies; Eq. (7) indicates that the materials at the disaster-stricken points are distributed in proportion to ensure the best local fairness; Eq. (8) is the unsatisfied rate of demand at the disaster-stricken points under various scenarios. Eq. (9) indicates that the difference between the unsatisfied rates of any two disaster-stricken points in all scenarios are within a given threshold to achieve the fairness of material distribution; Eq. (10) is the limit on the amount of emergency materials available; Eq. (11) is the capacity constraint of the distribution center; Eq. (12) is the non-negative integer constraint; Eq. (13) is the 0-1 variable constraint.

\subsection{Fuzzy parameter processing}

The demand of a disaster-stricken point is a triangular fuzzy number, and the expected value conversion is carried out using the fuzzy parameter expectation formula proposed in [16]. The expected demand for c-type materials at disaster-stricken point $i$ in the initial stage of post-earthquake rescue can be handled by Eq. (14).

$$
E\left(\tilde{D}_{i c}\right)=\frac{D_{i c}^{1}+2 D_{i c}^{2}+D_{i c}^{3}}{4}
$$

\section{Model solving}

Since the objective function and constraints in Eq. (4), Eq. (7), Eq. (8) are non-linear expressions, the model established is a non-linear 0-1 mixed integer programming model. The solution of this model is an NP-hard problem, and the branch and bound 


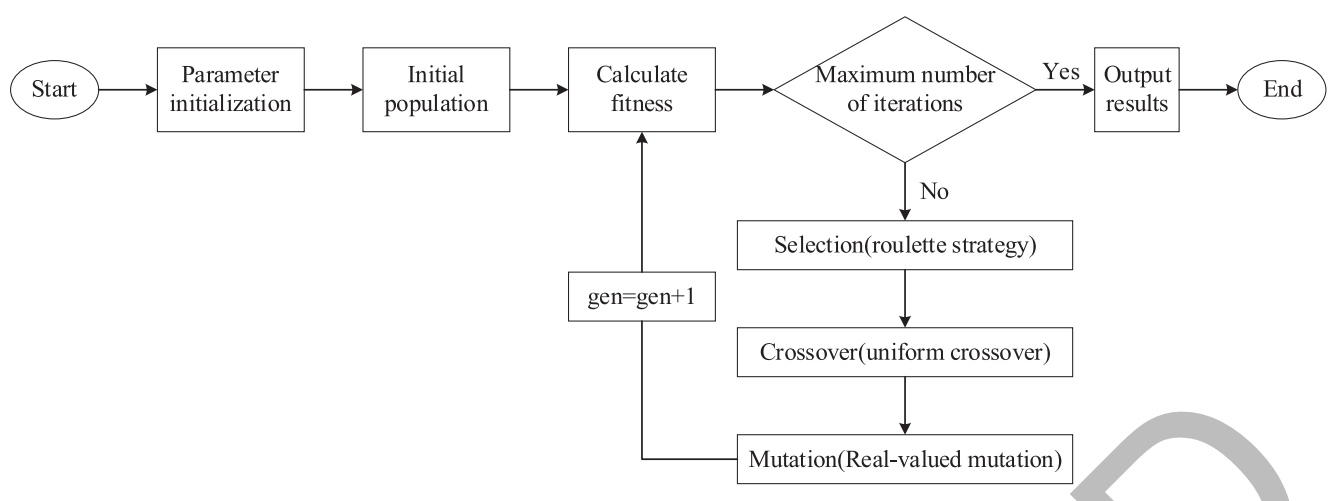

Fig. 1. Algorithm flow chart.

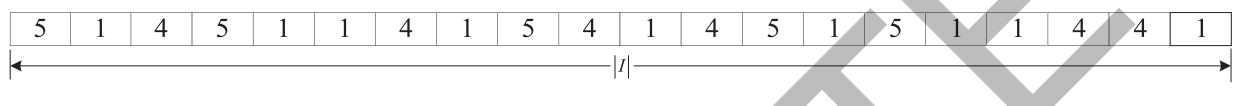

Fig. 2. Schematic diagram of chromosome coding.

algorithm or optimization tools such as Gurobi and Cplex cannot be used to accurately solve the problem. Therefore, according to the characteristics of the model, a hybrid genetic algorithm with integer coding is designed. The algorithm flow is shown in Fig. 1 .

(1) Parameter initialization

Initialize the population size popsize, maximum number of iterations max gen, crossover probability $p_{c}$, mutation probability $p_{m}$ and other parameters.

(2) Chromosome coding

The length of the chromosome is $|I|$. Take Fig. 2 as an example. Assuming that there are 7 candidate facilities and 20 disaster-stricken points in the network, the codes in Fig. 2 mean that open distribution centers are 1, 4, and 5; facility 1 serves disasterstricken points $2,5,6,8,11,14,16,17,20$; facility 4 serves disaster-stricken points $3,7,10,12,18,19$; facility 5 serves disaster-stricken points $1,4,9,13$, 15. Therefore, the location solution $X_{j}$ and allocation solution $Y_{i j}$ can be obtained directly through encoding and decoding. The supply $Q_{j c}, Q_{i c w}$ is decoded according to the variable $Y_{i j}$, and the total amount of materials from the designated distribution center is allocated in proportion according to the demand of the disaster-stricken point.

(3) Initialize the population

The initial population is randomly and uniformly generated based on the randi function embedded in matlab.

(4) Calculation of fitness value
The established model is a minimization problem. Here, the fitness value is rewritten as the reciprocal of the objective function value. Let Fitness (chromosome 1 (gen)) be the fitness value of the $i$ th chromosome in the genth generation and $Z_{i}(\mathrm{ge})$ be the objective function value of the first generation, then:

$$
\text { Fitness } \left._{(\text {chromosome }}(\text { gen })\right)=\frac{1 / Z_{i}(\text { gen })}{\sum_{i}\left(1 / Z_{i}(\text { gen })\right)}
$$

Use penalty function to deal with constraints (9) to avoid infeasible solutions.

(5) Genetic manipulation

(1) Choose. Use roulette for selection operations. At the same time, the elite retention strategy is added to copy the existing optimal individuals to the next generation to accelerate the convergence and enhance the effectiveness of the algorithm [17].

(2) Cross. The uniform crossover strategy is adopted: a 0-1 matrix with length $|I|$ and scale popsize is uniformly and randomly generated, and two chromosomes are randomly selected for crossover according to the corresponding position of the gene position. As shown in Fig. 3, if the corresponding position of the locus is 0 , select the gene of chromosome 1 ; otherwise, select the gene of chromosome 2; thus, two new chromosomes are obtained. 


\begin{tabular}{|c|c|c|c|c|c|c|c|c|c|}
\hline 6 & 4 & 1 & 7 & 3 & 2 & 1 & 2 & 3 & 7 \\
\hline 1 & 2 & 6 & 3 & 6 & 2 & 4 & 1 & 6 & 6 \\
\hline 0 & 1 & 0 & 0 & 1 & 1 & 1 & 0 & 1 & 0 \\
\hline 6 & 2 & 1 & 7 & 6 & 2 & 4 & 2 & 6 & 7 \\
\hline 1 & 4 & 6 & 3 & 3 & 2 & 1 & 1 & 3 & 6 \\
\hline
\end{tabular}

Fig. 3. Schematic diagram of chromosome crossover.

\begin{tabular}{|l|l|l|l|l|l|l|l|l|l|}
\hline 6 & 2 & 1 & 7 & 6 & 2 & 4 & 2 & 6 & 7 \\
\hline 1 & 4 & 6 & 3 & 3 & 2 & 1 & 1 & 3 & 6 \\
\hline 6 & 2 & 7 & 7 & 6 & 5 & 4 & 2 & 6 & 7 \\
\hline 1 & 1 & 6 & 3 & 3 & 2 & 1 & 1 & 4 & 6 \\
\hline
\end{tabular}

Fig. 4. Schematic diagram of chromosome mutation.

(3) Mutation. As shown in Fig. 4, two genes of the chromosome are randomly selected for realvalued mutation. In order to prevent the gene value after mutation from exceeding the critical value, an area scanner is added. The variable mutation rate is adopted, that is, a larger value is adopted for $p_{m}$ at the beginning of the algorithm iteration to promote the diversity of the solution; after a certain number of iterations, a smaller value is adopted for $p_{m}$ to accelerate the convergence [17].

(6) Algorithm termination conditions If the iteration algebra reaches max gen, the algorithm terminates and calculates the optimal solution.

\section{Case analysis}

\subsection{Analysis of calculation results}

Take the 5.12 Wenchuan Earthquake as a case for analysis. Assuming that the disaster-stricken points are 30 hardest-hit areas, the distance between the disaster-stricken points is calculated by coordinate positions. The coordinate information and material demand information of the 30 hardest-hit areas can be found in [18]. There are 3 types of main emergency supplies, and the current available quantities of the 3 types of emergency supplies are 26,000 units, 22,500 units and 6,400 units, respectively. The coordinates and processing capacity information of 15 candidate distribution centers are shown in Table 1 . The parameter values of the transportation distance, normal driving time, road condition coefficient, and single transportation capacity limit from each candidate distribution center to the disaster-stricken point are shown in [18]. Assuming that there are 4 disruption scenarios, as shown in Table 2. In each of scenarios 2 to 4 , one facility was interrupted. Other parameter settings were set as follows, $v_{i j}=80 \mathrm{~km} / \mathrm{h}, \xi=0.05$.

The genetic algorithm parameters were set as follows $[17,19]$, popsize $=100, \max$ gen $=400, p_{c}=$ 0.9 . For the first 100 iterations, $p_{m}$ took 0.2 ; after 100 generations, $p_{m}$ took 0.1. Matlab $2010 \mathrm{~b}$ was used as the programming language and implementation platform, the program was run on a mobile workstation with Intel Core i5 CPU and $8 \mathrm{G}$ memory, and the opti-

Table 1

Information of candidate distribution center

\begin{tabular}{|c|c|c|c|c|c|c|c|}
\hline No. & $X$ coordinate & oordinate & $\begin{array}{c}\text { Maximum } \\
\text { processing } \\
\text { ability }\end{array}$ & No. & $X$ coordinate & $Y$ coordinate & $\begin{array}{c}\text { Maximum } \\
\text { processing } \\
\text { ability }\end{array}$ \\
\hline 1 & 19.7 & 15 & 14000 & 9 & 28.8 & 13.2 & 12700 \\
\hline 2 & 11.5 & 24.7 & 13000 & 10 & 23.3 & 23.6 & 11000 \\
\hline 3 & 16 & 20.8 & 11000 & 11 & 10.2 & 19.7 & 13000 \\
\hline 4 & 23.7 & 11.7 & 12500 & 12 & 11.7 & 24.5 & 13300 \\
\hline 5 & 23.2 & 14.1 & 13000 & 13 & 26.4 & 17.6 & 12700 \\
\hline 6 & 11 & 18.9 & 12300 & 14 & 18.6 & 19.3 & 13000 \\
\hline 7 & 27.3 & 9.2 & 12500 & 15 & 26.4 & 9.4 & 11000 \\
\hline 8 & 14.4 & 21.3 & 13000 & - & - & - & - \\
\hline
\end{tabular}

Table 2

Disruption scenario set of candidate distribution centers

\begin{tabular}{|c|c|c|c|c|c|c|c|c|c|c|c|c|c|c|c|}
\hline Scenario/facility & 1 & 2 & 3 & 4 & 5 & 6 & 7 & 8 & 9 & 10 & 11 & 12 & 13 & 14 & 15 \\
\hline Scenario1 & 1 & 1 & 1 & 1 & 1 & 1 & 1 & 1 & 1 & 1 & 1 & 1 & 1 & 1 & $\overline{1}$ \\
\hline Scenario2 & 1 & 0 & 1 & 1 & 1 & 1 & 1 & 1 & 1 & 1 & 1 & 1 & 1 & 1 & 1 \\
\hline Scenario3 & 1 & 1 & 1 & 0 & 1 & 1 & 1 & 1 & 1 & 1 & 1 & 1 & 1 & 1 & 1 \\
\hline Scenario4 & 1 & 1 & 1 & 1 & 1 & 1 & 1 & 1 & 0 & 1 & 1 & 1 & 1 & 1 & 1 \\
\hline
\end{tabular}

Note: 0 means that the facility is interrupted. Data source: reference [18]. 


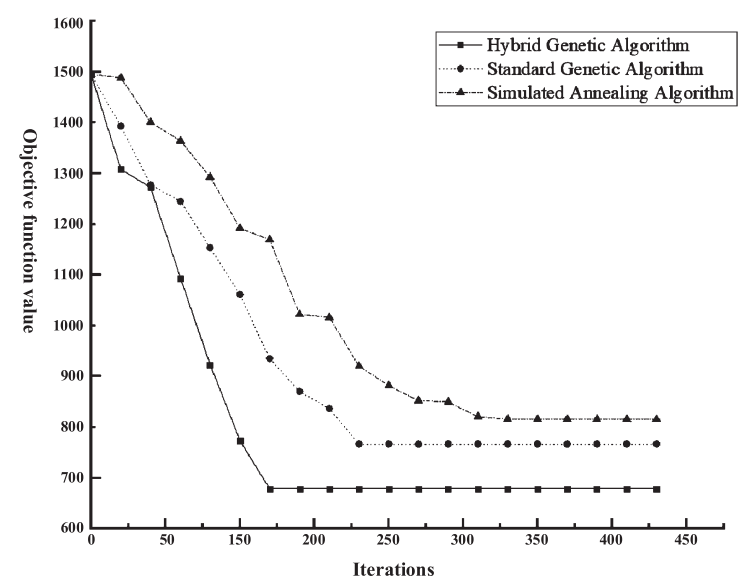

Fig. 5. Comparison of the iteration processes of different algorithms.

mal solution of 10 calculation results was taken as the final solution. The algorithm convergence time was $213.77 \mathrm{~s}$, the algorithm convergence curve is shown in Fig. 5, and the calculation results are shown in Table 3.

In order to test the reliability of the built model, comparison was made between the current optimization scheme (denoted as scheme 1) with the optimization scheme (denoted as scheme 2 and the results are shown in Table 4) that did not consider facility disruption scenarios. In scheme 2 , the facility disruption scenario was reconsidered, the target value under each scenario was calculated, and the result of scheme 1 was compared (see Table 5). The results showed that under scenario 1 , scheme 2 was slightly better than scheme1 (a gap of $-8.3 \%$ ); while in other scenarios, scheme 1 was significantly better than scheme 2 (a gap greater than $21.6 \%$ ). This meant that once a disruption occurred, there would be a lot of emergency costs. Therefore, considering the disruption scenario in the emergency logistics network design stage could improve the reliability and robustness of the system.

\subsection{Comparison and evaluation of the algorithms}

In order to prove the applicability and effectiveness of the hybrid genetic algorithm with integer coding proposed in this research, comparison is performed on the proposed algorithm, simulated annealing algorithm and standard genetic algorithm. In the simulated annealing algorithm, the parameters are set as follows: initial temperature of $1000^{\circ} \mathrm{C}$, end temperature of $0.05^{\circ} \mathrm{C}$, temperature reduction coefficient of 0.8 , and step size of 5 , It is stipulated that the iteration can be stopped when the temperature reaches $0.5^{\circ} \mathrm{C}$, and the maximum number of iterations is 1000 . In the genetic algorithm, the parameter setting is the same as that of the algorithm designed in this study. According to Fig. 5, the performance of the hybrid genetic algorithm and the standard genetic algorithm is much better than the simulated annealing algorithm in the early iteration process (as indicated by the decline rate). Besides, the hybrid genetic algorithm is superior to the other two algorithms, due to

Table 3

\begin{tabular}{lccccccccc}
\multicolumn{7}{c}{ The location-allocation solution considering facility disruption scenarios } \\
\hline$X_{j}$ & $Y_{i j}$ & $Q_{j 1}$ & $Q_{j 2}$ & $Q_{j 3}$ & $X_{j}$ & $Y_{i j}$ & $Q_{j 1}$ & $Q_{j 2}$ & $Q_{j 3}$ \\
\hline 1 & $2,3,8,10,20$ & 5366 & 4645 & 1321 & 11 & 22,29 & 308 & 266 \\
3 & 23 & 206 & 178 & 50 & 12 & $25,27,28$ & 368 & 319 & 91 \\
5 & 21 & 2682 & 2321 & 660 & 13 & $7,11,14,19$ & 4834 & 4182 & 1189 \\
6 & $1,5,24,26,30$ & 2982 & 2581 & 734 & 14 & 4 & 302 & 261 & 74 \\
7 & 13 & 1144 & 990 & 282 & 15 & 12 & 2770 & 2398 \\
10 & $6,9,15,16,17,18$ & 5038 & 4359 & 1241 & & & 682 \\
\hline
\end{tabular}

Table 4

Location-allocation solutions without considering facility disruption scenarios

\begin{tabular}{|c|c|c|c|c|c|c|c|c|c|}
\hline$X_{j}$ & $Y_{i j}$ & $Q_{j 1}$ & $Q_{j 2}$ & $Q_{j 3}$ & $X_{j}$ & $Y_{i j}$ & $Q_{j 1}$ & $Q_{j 2}$ & $Q_{j 3}$ \\
\hline 1 & $2,3,8,10,20$ & 5366 & 4645 & 1321 & 9 & $12,13,19$ & 5383 & 4658 & 1325 \\
\hline 2 & $25,27,28$ & 368 & 319 & 91 & 10 & $6,9,15,17,18$ & 3764 & 3257 & 927 \\
\hline 3 & 23 & 206 & 178 & 50 & 11 & 22,29 & 308 & 266 & 75 \\
\hline 4 & 5,21 & 3936 & 3407 & 969 & 13 & $7,11,14,16$ & 4639 & 4014 & 1142 \\
\hline 6 & $1,24,26,30$ & 1727 & 1495 & 425 & 14 & 4 & 302 & 261 & 74 \\
\hline
\end{tabular}


Table 5

Comparison of two optimization schemes

\begin{tabular}{lccccccc}
\hline Scenario no. & Scheme1 & Scheme2 & gap (\%) & Scenario no. & Scheme1 & Scheme2 & gap $(\%)$ \\
\hline Scenario1 & 793.4 & 727.8 & -8.3 & Scenario3 & 793.4 & 1062.5 & 33.9 \\
Scenario2 & 793.4 & 1051.8 & 32.6 & Scenario4 & 793.4 & 965.1 & 21.6 \\
\hline
\end{tabular}

smaller number of iterations, less running time and smaller value of the objective function.

\section{Conclusion}

The initial post-earthquake stage is a golden period for emergency rescue. During this period, the demand for emergency supplies is large and the urgency is at high level. At this time, the establishment of a rapid and effective emergency logistics network is of great value to rescue and disaster relief. Considering that in the post-earthquake emergency rescue logistics system, emergency facilities are exposed to secondary disasters such as aftershocks, which are not completely reliable. Therefore, the design of an emergency logistics network needs to consider the phenomenon of facility disruption. Since the specific probability of facility disruption can hardly be accurately obtained, the method of setting typical disruption scenarios has better practicality. On this basis, based on the characteristics of post-earthquake rescue in the initial stage, this paper establishes an emergency facility location selection that considers facility disruption scenarios, multiple types of ambiguous requirements, facility processing capacity limitations, fair distribution of materials, and partial damage to the transportation network-distribution model. A hybrid genetic algorithm is designed based on integer coding. Finally, the model and algorithm are verified by taking the 5.12 Wenchuan Earthquake for case analysis.

Numerical simulation results show that if the facilities are interrupted after the earthquake, the results of this paper can optimize the system target by more than $21.6 \%$; even if there is no emergency facility disruption after the earthquake, the difference between the optimization result and the optimal target value is only $8.3 \%$. Compared with standard genetic algorithm and simulated annealing algorithm, this hybrid genetic algorithm has good convergence. Therefore, the main research conclusion is obtained as follows. After considering the facility disruption scenario, even if some facilities fail, the entire emergency logistics network can still operate well. The optimization result has certain reliability and robustness. There- fore, it is necessary to consider the disruption risk of facilities in the system design stage and establish a more reliable and robust emergency logistics network.

Further research can take into account the emergency material collection and distribution points, and construct an optimization design problem for multi-level emergency logistics network considering facility disruption scenarios. It is also possible to comprehensively consider the staged characteristics in the later stage of rescue, and study the dynamic location-allocation of multiple types of emergency facilities under disruption scenarios.

\section{Acknowledgments}

This study was supported by Key R \& D projects of the Ministry of Science and Technology of China (863 Program) (Grant No.2018YFB1601400), Key R \& D Project of Sichuan Science and Technology Department (Grant No.2019YFG0001) and China Railway Group Co., Ltd. (Grant No. KF2019-010-B)

\section{Data announcements}

In the case analysis part of this study, the location and material requirements of the 30 hardest-hit areas after the 5.12 Wenchuan Earthquake in China were used. In order to facilitate the modeling and algorithm implementation of this study, we abstracted the location data in the form of coordinates. Professor Jin Zhang once cooperated with Chongqing Technology and Business University of China in the Sichuan Social Science Eleventh Five-Year Plan project (2010-2016). The project was to study the distribution of emergency supplies under unconventional emergencies based on the Wenchuan Earthquake. A large amount of data was obtained in the research, but the subject had signed a confidentiality agreement. The research data of the subject cannot be made public due to the restrictions of the Chinese government policy. The relevant data we used came from reference [18], which is a master's degree thesis. Part of the data used in this thesis was extracted from the above project, 
and we are happy to give the address (in Chinese) of the thesis:

https://kns.cnki.net/KCMS/detail/detail.aspx?db code $=$ CMFD $\&$ dbname $=$ CMFD201802 $\&$ filename $=$ 1018218985.nh\&v=MTk5Mjc1RnRqRXFwRWJQ SVI4ZVgxTHV4WVM3RGgxVDNxVHJXTTFGc kNVUjdxZllPUnZGaUhsVnIvQ1ZGMjZGckc=

\section{Authors' contribution}

Junxiang $\mathrm{Xu}$ is the first author of this paper. He is mainly responsible for writing the core part, researching emergency facility location-allocation modeling and algorithm design. Jingni Guo is the second author of this paper. She is mainly responsible for the writing of the calculation part of this paper. Jin Zhang is the third author of this paper. He is a doctoral supervisor, and he is mainly responsible for directing the theoretical research of this paper and algorithm design, and providing financial support for this research. Yongdong sun is mainly responsible for the English language editing of the full text. Weihua Liu and Hui Ma are technical engineers of China Railway No.2 Engineering Group Co., Ltd. They provide case analysis guidance for our research.

\section{References}

[1] F.S. Salman and E. Yücel, Emergency facility location under random network damage: insights from the Istanbul case [J], Computers and Operations Research 62(10) (2015), 266-281.

[2] C. Boonmee, M. Arimura and T. Asada, Facility location optimization model for emergency humanitarian logistics [J], International Journal of Disaster Risk Reduction 24(9) (2017), 485-498.

[3] G.H. Tzeng, H.J, Cheng and T.D. Huang, Multi-objective optimal planning for designing relief delivery systems[J], Transportation Research Part E: Logistics and Transportation Review 43(6) (2007), 673-686.

[4] X. Xiong, F. Zhao, Y. Wang, et al., Research on the Model and Algorithm for Multimodal Distribution of Emergency Supplies after Earthquake in the Perspective of Fairness[J], Mathematical Problems in Engineering 2019 (2019), 1-12.

[5] G. Zhang, J. Shi, S.S. Chaudhry, et al., Multi-period multiproduct acquisition planning with uncertain demands and supplier quantity discounts[J], Transportation Research Part E: Logistics and Transportation Review 132 (2019), 117-140.
[6] M. Yahyaei and A. Bozorgi-Amiri, Robust reliable humanitarian relief network design: an integration of shelter and supply facility location[J], Annals of Operations Research 2018, 1-20.

[7] D. Shishebori and A.Y. Babadi, Robust and reliable medical services network design under uncertain environment and system disruptions[J], Transportation Research Part E 77(5) (2015), 268-288.

[8] R. Mishra, A.K. Pundir and L. Ganapathy, Assessment of manufacturing flexibility[J], Management Research Review 37(8) (2014), 750-776.

[9] L.V. Snyder and M.S. Daskin, Reliability models for facility location: the expected failure cost case[J], Transportation Science 39(3) (2005), 400-416.

[10] Y. An, B. Zeng, Zhang, et al., Reliable p-median facility location problem: two-stage robust models and algorithms [J], Transportation Research Part B: Methodological 64(6) (2014), 54-72.

[11] M. Albareda-Sambola, Y. Hinojosa and J. Puerto, The reliable p-median problem with at-facility service[J], European Journal of Operational Research 245(3) (2015), 656-666.

[12] M. Sadeghi, J.F. Tavakkoli-Moghaddam, et al., A new stochastic approach for a reliable p-hub covering location problem [J], Computers and Industrial Engineering 90(12) (2015), 371-380.

[13] N. Aydin and A. Murat, A swarm intelligence based sample average approximation algorithm for the capacitated reliable facility location problem[J], International Journal of Production Economics 145(1) (2013), 173-183.

[14] Y.F. Zhou, Z.J. Ma and M.W.K., A Reliability P-median Location Model for Relief Supplies Reserve Bases[J], Management Review 27(5) (2015), 198-208.

[15] D. Fu, Y.Z. Chen and X. Wan, The study on the reliable model for the regional emergency storage facility under the risk of natural disaster[J], Operations Research and Management Science 24(3) (2015), 14-19.

[16] H. Garg, A novel approach for analyzing the reliability of series-parallel system using credibility theory and different types of intuitionistic fuzzy numbers [J], Journal of the Brazilian Society of Mechanical Sciences and Engineering 38(3) (2016), 1021-1035.

[17] Y.F. Zhou, Z. Li and S.F. Liu, Location-inventory Model of National Blood Strategic Reserves based on - robust Stochastic Optimization Method[J], Chinese Journal of Management Science 26(10) (2018), 52-63.

[18] N. Chen, Research on Reliability Optimization of PostEarthquake Emergency Logistics Network under Facility Disruption Scenarios[D], Chongqing Technology and Business University, 2018.

[19] X. Huang and L. Song, An emergency logistics distribution routing model for unexpected events[J], Annals of Operations Research 269(6) (2018), 1-17. 